\title{
MODELO DE EQUILIBRIO GENERAL COMPUTABLE PARA NUEVO LEÓN: ANÁLISIS DEL PROGRAMA DE APOYO DIRECTO AL ADULTO MAYOR
}

\section{COMPUTABLE GENERAL EQUILIBRIUM MODEL FOR NUEVO LEON: ANALYSIS OF DIRECT SUPPORT PROGRAM FOR THE ELDERLY}

Esther Guadalupe Santos Vázquez Universidad Autónoma de Nuevo León, México esther.sv21@gmail.com
Joana Cecilia Chapa Cantú

Universidad Autónoma de Nuevo

León, México

joana.chapacn@uanl.edu.mx

\section{RESUMEN}

Como objetivo general, se estima un modelo de equilibrio general computable (MEGC) para el estado de Nuevo León, que permite determinar los efectos de políticas públicas en la entidad, al tomar en cuenta las interrelaciones entre familias, empresas, gobierno y sector externo. Como objetivo específico, se analizan los efectos del Programa de apoyo directo al adulto mayor (PAM) sobre los niveles y distribución del consumo, producción e ingreso de Nuevo León.

Se modela la economía del estado y para ello se asume competencia perfecta. Esta entidad federativa se considera como una economía regional con un sector externo agregado (resto de las entidades federativas del país y el resto del mundo). En esta economía interactúan: diez tipos de hogares, diferenciados por el decil de ingreso; 17 sectores productivos o empresas; 
dos niveles de gobierno, local y federal y un sector externo agregado. Se producen y comercian: 17 productos intermedios, diez bienes de consumo final y un bien de inversión agregado. Las familias ofrecen 17 tipos de trabajo y un tipo de capital. El modelo es estático y de tipo neoclásico.

Entre los resultados, se obtiene que el PAM ha tenido los efectos redistributivos buscados por la autoridad estatal, ya que por medio del coeficiente de Gini se estima que ese programa redistribuye el ingreso de manera más equitativa. Adicionalmente, se han generado ganancias de eficiencia en la producción, el ingreso y el consumo privado del estado de Nuevo León al transferir los recursos al PAM, contra que los gaste el gobierno de la forma que dicta su estructura de presupuesto.

Palabras clave: economía regional, política pública, equilibrio general, distribución del ingreso.

Clasificación JEL: C68, H54

\begin{abstract}
The general objective of this paper is to estimate a Computable General Equilibrium Model (CGE) for the state of Nuevo Leon, Mexico, to analyze the effects of public policies on this regional economy taking into account the interrelationships between households, industries, government and external sector. As an empirical application, we analyze the effects on consumption, production and income distribution of a direct transfer to older poor people named: Programa de Apoyo Directo al Adulto Mayor (PAM). We model this regional economy assuming perfect competition. In this economy interact: 10 households differentiated by their income level, 17 productive sectors, two levels of government, local and federal, and aggregated external sector. In this economy are traded: 17 intermediates goods, 10 final goods and an investment good. Families provide 17 types of labor and one kind of capital. The model is static and neoclassical type.

The main results suggest that the program has had the redistributive effects desired by the government, since is estimated through the Gini coefficient
\end{abstract}


that this program redistributes income more equitably. Additionally, transferring resources through PAM generates efficiency gains on production, income and households consumption in the state of Nuevo Leon, compared to spending such resources following the traditional government budget.

Keywords: Regional economy, public policy, general equilibrium, income distribution. 



\section{INTRODUCCIÓN}

A finales del siglo XIX el economista francés León Walras planteó una línea de investigación conocida como la teoría del equilibrio general, la cual, con el paso de los años, se fue perfeccionando a través de una gran cantidad de aportaciones teóricas, matemáticas y computacionales. A finales de la década de los setenta fue posible llevar al campo práctico esta noción, se construyeron con datos de economías reales los llamados modelos de equilibrio general computable (MEGC). Modelos que se caracterizan porque permiten determinar el efecto de las políticas públicas sobre las decisiones económicas de las familias, empresas, gobierno y sector externo; en un ambiente de interdependencia general.

En México se han construido MEGC en el ámbito nacional para estudiar temas tan diversos como: la reforma fiscal de los ochenta (Serra, 1979; Kehoe y Serra,1983; Kehoe, Serra y Solís, 1986 en bibliografía); la crisis de la deuda (Kehoe y Serra, 1991); la firma del Tratado de Libre Comercio de América del Norte (Sobarzo, 1991, 1994; Chapa, 2003); las reformas agrarias realizadas a raíz de este tratado (Boyd y Dooroodian, 1999; Harris, 2000); y la introducción de programas como Progresa (Coady y Harris, 2004) ${ }^{1}$.

La construcción de MEGC para el ámbito regional es más escasa. El problema principal es la falta de información oficial en ese grado de desagregación. Los antecedentes que existen de modelos regionales son en su mayoría para las comunidades españolas de Andalucía (Cardenete y Sancho, 2003; André, Cardenete y Velázquez, 2005; Cardenete, Hewings y Velázquez, 2006), Extremadura (de Miguel y Manresa, 2008; Cardenete, de Miguel y Pérez, 2009) y Cataluña (Llop y Manresa, 2004). En cuanto a México, los trabajos realizados para regiones o estados son muy pocos. González y Sobarzo (1999) construyeron un modelo para Jalisco de tipo keynesiano; mientras que Hampton, Taylor y Yúnez (1999) y Dyer, Taylor y Yuñez (1999) realizaron unos MEGC para algunas zonas rurales de Michoacán, con datos levantados directamente, con los que analizan los efectos del programa federal Procampo.

\footnotetext{
${ }^{1}$ Progresa cambió de nombre a Oportunidades en el año 2002.
} 
En el presente trabajo se elabora un MEGC para el estado de Nuevo León, el cual constituye uno de los modelos pioneros en México a nivel regional, así como para esta entidad. El modelo está diseñado para analizar los efectos de políticas sobre la redistribución del ingreso, ya que diferencia a las familias por deciles de ingreso. Además, con la finalidad de mostrar su aplicación, se analizan los efectos sobre la economía de Nuevo León de una de las políticas sociales más importantes de la última década: el Programa de apoyo directo al adulto mayor (PAM).

El trabajo se organiza de la siguiente forma: en la sección dos se profundiza sobre el programa analizado; en la tres se detalla el modelo; en la cuarta se describe la base de datos utilizada para alimentar el modelo; en la sección cinco se presentan los resultados y la última contiene las conclusiones.

\section{PROGRAMA DE APOYO DIRECTO AL ADULTO MAYOR}

Los adultos mayores son un grupo vulnerable que ha captado la atención del gobierno federal y varios gobiernos estatales debido a diversas razones, entre ellas, la gran cantidad de personas no afiliadas a los sistemas de seguridad social que, probablemente, no contarán con ingresos por pensión durante su vejez; el envejecimiento de la población que se espera para los próximos años y los antecedentes históricos de mayores niveles de pobreza entre las personas de la tercera edad.

En México existen programas focalizados, tanto federales como estatales, para combatir la pobreza entre los adultos mayores. En el caso de los primeros se encuentra el programa Oportunidades, iniciado en 1997, pero que desde el año 2006 cuenta con un componente de apoyo monetario para las personas de la tercera edad que sean integrantes de una familia beneficiaria; otro es el programa 70 y Más, puesto en marcha en 2006 y que otorga una transferencia de 500 pesos mensuales (entregados bimestralmente) a las personas mayores de 70 años que no son beneficiarias de Oportunidades y viven en localidades con un máximo de 30 mil habitantes.

En cuanto a los gobiernos estatales, como se observa en el cuadro 1, son alrededor de nueve entidades las que han instrumentado programas de apoyo a los adultos mayores mediante transferencias directas. 


\section{Cuadro 1}

Programas estatales de apoyo a los adultos mayores

\begin{tabular}{|c|c|c|c|}
\hline Estado & Nombre del programa & $\begin{array}{l}\text { Año de inicio } \\
\text { del programa }\end{array}$ & $\begin{array}{c}\text { Transferencia } \\
\text { mensual }\end{array}$ \\
\hline Chihuahua & Vive a Plenitud & 2005 & $\$ 862.00$ \\
\hline Distrito Federal & $\begin{array}{c}\text { Pensión Alimentaria para los } \\
\text { Adultos Mayores }\end{array}$ & 2003 & $897.30 *$ \\
\hline Guerrero & $\begin{array}{c}\text { Pensión Guerrero Para Vivir } \\
\text { Mejor (PROVIVIR) }\end{array}$ & 2003 & $\$ 400.00$ \\
\hline Jalisco & $\begin{array}{c}\text { Atención a los Adultos } \\
\text { Mayores }\end{array}$ & 2007 & $\$ 500.00$ \\
\hline Nuevo León & $\begin{array}{c}\text { Programa de Apoyo Directo al } \\
\text { Adulto Mayor }\end{array}$ & 2004 & $\$ 700.00$ \\
\hline Tlaxcala & $\begin{array}{l}\text { Con nuestros mayores, un } \\
\text { nuevo compromiso }\end{array}$ & 2006 & $\$ 428.50 *$ \\
\hline Veracruz & $\begin{array}{l}\text { Pensión Alimenticia para } \\
\text { Adultos Mayores }\end{array}$ & 2005 & $\$ 850.00 *$ \\
\hline Yucatán & Reconocer & 2007 & $\$ 550.00$ \\
\hline Zacatecas & Rescate a los Abuelos & 2005 & $\$ 400.00$ \\
\hline
\end{tabular}

Nota:*estimaciones para 2011.

Fuente: Elaboración propia con información de las páginas oficiales de los gobiernos estatales.

En el caso de Nuevo León, desde el año 2004 se instituyó el Programa de apoyo directo al adulto mayor, dirigido a las personas mayores de 70 años. Inicialmente se entregaba una transferencia de 500 pesos mensuales, depositados en una tarjeta electrónica (zona urbana) o por medio de vales (zona rural) utilizables en una gran variedad de tiendas de autoservicio; sin embargo, a partir de febrero de 2010, el apoyo es de 700 pesos mensuales.

En lo que respecta a los beneficiarios, cuando se diseñó el PAM se contemplaron a las personas que tuvieran 70 años o más, originarias del estado de Nuevo León o residentes por al menos 5 años, que no contaran con ingresos por pensión o jubilación, ni con apoyos gubernamentales y, en general, con nivel socioeconómico bajo. En un principio se impuso como requisito el vivir en alguno de los 53 polígonos de pobreza del área 
metropolitana de Monterrey (AMM) o de los 15 polígonos de pobreza ubicados en cabeceras municipales del resto del estado, no obstante, el programa logró cubrir por completo esta población, que inicialmente se estimaba en 20517 adultos mayores (Martínez, 2006), por lo que el apoyo se extendió a localidades de todos los municipios. ${ }^{2}$

Como se observa en el cuadro 2, en el año 2004, 81.47\% del apoyo otorgado por el programa se concentró en el primer decil de ingreso, con el paso del tiempo su distribución ha sido más adecuada a los objetivos del programa ya que en $2010,88.78 \%$ de la transferencia se dirigió hacia el grupo más pobre.

\section{Cuadro 2}

Distribución porcentual por deciles del PAM

\begin{tabular}{cccc}
\hline Decil & $\begin{array}{c}\text { Intervalo de ingreso } \\
\text { trimestral * }\end{array}$ & $\begin{array}{c}\text { Distribución } \\
\text { porcentual del PAM } \\
\text { en 2004** }\end{array}$ & $\begin{array}{c}\text { Distribución } \\
\text { porcentual del PAM } \\
\text { en 2010*** }\end{array}$ \\
\hline $\mathbf{1}$ & $807-10807$ & $81.47 \%$ & $88.78 \%$ \\
$\mathbf{2}$ & $10810-14803$ & $7.10 \%$ & $4.38 \%$ \\
$\mathbf{3}$ & $14815-18440$ & $4.69 \%$ & $2.60 \%$ \\
$\mathbf{4}$ & $18454-22200$ & $2.54 \%$ & $1.48 \%$ \\
$\mathbf{5}$ & $22208-26984$ & $1.86 \%$ & $1.07 \%$ \\
$\mathbf{6}$ & $26984-32187$ & $1.11 \%$ & $0.64 \%$ \\
$\mathbf{7}$ & $32211-39608$ & $0.64 \%$ & $0.43 \%$ \\
$\mathbf{8}$ & $39615-50133$ & $0.36 \%$ & $0.21 \%$ \\
$\mathbf{9}$ & $50174-72767$ & $0.21 \%$ & $0.19 \%$ \\
$\mathbf{1 0}$ & $72851-650123$ & $0.02 \%$ & $0.22 \%$ \\
\hline
\end{tabular}

Fuente: * Aguayo, Chapa, Ramírez y Rangel (2009); ** Chapa, Rangel y Ramírez (2008); *** elaboración propia con los resultados de la Encuesta de características socioeconómicas del adulto mayor realizada al Padrón de beneficiarios del programa.

\footnotetext{
${ }^{2}$ Un polígono de pobreza es un espacio social rezagado respecto al desarrollo alcanzado por el resto de la metrópoli donde está situado (Gómez, Martínez y Treviño, 2009).
} 


\section{MODELO}

Se modela la economía del estado, para ello se asume competencia perfecta, es decir, todos los agentes económicos toman sus decisiones considerando los precios como dados. El estado de Nuevo León se considera como una economía regional con un sector externo agregado (resto de las entidades federativas del país y el resto del mundo). En esta economía interactúan: diez tipos de hogares, diferenciados por el decil de ingreso; 17 sectores productivos o empresas; dos niveles de gobierno, local y federal y un sector externo agregado. Se producen y comercian: 17 productos intermedios, diez bienes de consumo final y un bien de inversión agregado. Las familias ofrecen 17 tipos de trabajo y un tipo de capital.

\subsection{Hogares}

Los hogares del decil de ingreso $h$ toman sus decisiones siguiendo un proceso de optimización en dos niveles. En el primer nivel, eligen el consumo agregado $\left(C_{h}\right)$ y el ahorro $\left(S_{h}\right)$, maximizan su utilidad sujeto a su ingreso disponible $\left(I D_{h}\right)$. Se asume que las funciones de utilidad son del tipo Cobb Douglas homogéneas de grado 1, y que los hogares toman los precios del bien de consumo agregado $\left(P A C_{h}\right)$ y del ahorro $(P S)$ como dados:

$$
\begin{gathered}
\operatorname{Max} U_{h}=\left(C_{h}\right)^{\beta_{h}}\left(S_{h}\right)^{1-\beta_{h}} \\
\text { s.a. } I D_{h}=P A C_{h} * C_{h}+P S * S_{h} \\
\text { para } h=1,2,3, \ldots, 10 .
\end{gathered}
$$

Donde el subíndice $h$ toma el valor de 1 si se trata del primer decil de ingreso, el valor de 2 para el segundo decil, y así sucesivamente hasta el valor 10, que lo toma el decil más rico. Nótese que cada familia enfrenta un nivel propio de precios del bien de consumo agregado, ya que estos se calculan al ponderar los precios de los bienes finales según los patrones de gasto, como se detallará más adelante en el apartado de precios. 
De tal manera que las elecciones óptimas de consumo y ahorro agregados están en función del ingreso disponible $\left(I D_{h}\right)$ y de los precios:

$$
\begin{gathered}
C_{h}=\frac{\beta_{h} * I D_{h}}{P A C_{h}} \\
S_{h}=\frac{\left(1-\beta_{h}\right) * I D_{h}}{P S}
\end{gathered}
$$

En el siguiente nivel eligen cuánto consumir de cada bien final $\left(c_{f, h}\right)$ minimizando el gasto total en consumo, dados los precios de dichos bienes $\left(P_{f}\right)$, sujeto al nivel de consumo agregado que fue óptimo en el primer nivel $\left(C_{h}\right)$. Se asume que el consumo total del hogar $h$ es un agregado de bienes finales, con una forma funcional del tipo Cobb Douglas homogénea de grado 1. Tal que, el proceso de optimización en el segundo nivel es:

$$
\begin{gathered}
\operatorname{Min} \sum_{f=1}^{10} P_{f} * c_{f, h} \\
\text { s.a. } C_{h}=A 0_{h} \prod_{f=1}^{10} c_{f, h}{ }^{\alpha_{f, h}} \\
\text { para } h=1,2,3, \ldots, 10 y f=1,2,3, \ldots, 10 .
\end{gathered}
$$

Donde $0 \leq \alpha_{f, h}<1, \sum_{f=1}^{10} \alpha_{f, h}=1$ y $A 0_{h}$ es el coeficiente de la función de consumo agregado del hogar con nivel de ingreso $h$.

El subíndice $f$ identifica a los bienes finales, los cuales son diez en total (ver cuadro 3). De esta manera, los niveles óptimos de consumo en bienes finales son:

$$
c_{f, h}=\left(\frac{\alpha_{f, h} C_{h}}{P_{f}}\right) * P A C_{h}
$$

El ingreso total de los hogares proviene del pago que reciben por ser los dueños de los factores productivos, trabajo $\left(L F_{h, l}\right)$ y capital $\left(K F_{h}\right)$; de 
las transferencias que reciben de ambos niveles de gobierno $\left(T R_{h, g}\right)$ y de los ingresos provenientes del sector externo $\left(R E M_{h}\right)$.

$$
\begin{gathered}
I T_{h}=\sum_{l=1}^{17}\left(P L_{l} * L F_{h, l}\right)+P K * K F_{h}+\sum_{g=1}^{2} T R_{g, h}+R E M_{h} \\
\quad \text { para } h=1,2,3, \ldots, 10 ; l=1,2,3, \ldots, 17 ; y g=1,2 .
\end{gathered}
$$

Donde $l$ denota los tipos de ocupación, que son un total de 17 en el modelo (ver cuadro 3 ). El subíndice $g$ identifica al nivel de gobierno, toma el valor de 1 para los gobiernos locales (estatal y municipal) y 2 para el gobierno federal. Por otro lado, $P L_{l}$ es el salario pagado al tipo de ocupación l; $P K$ es la renta pagada al capital y $R E M_{h}$ son las remesas provenientes del extranjero recibidas por el hogar del decil de ingreso $h$.

Las transferencias que otorgan los gobiernos locales $\left(T R_{1, h}\right)$ consisten en el programa de Apoyo directo al adulto mayor $\left(P A M_{h}\right)$, el programa de Apoyo a personas con discapacidad $\left(D I S_{h}\right)$, así como becas y ayudas $\left(B Y A_{h}\right)$; mientras que las transferencias que da el gobierno federal $\left(T R_{2, h}\right)$ son las del programa Oportunidades.

$$
T R_{1, h}=P A M_{h}+D I S_{h}+B Y A_{h}
$$

Las familias contribuyen al sector público al pagar un impuesto al ingreso por la venta de los factores productivos, trabajo y capital, a cada nivel de gobierno $g\left(\mathrm{TH}_{g, h}\right)$; por lo tanto, el ingreso disponible es:

$$
\begin{gathered}
I D_{h}=\left(1-\sum_{g=1}^{2} T H_{g, h}\right) * I G_{h}+\sum_{g=1}^{2} T R_{g, h} \\
+R E M_{h}
\end{gathered}
$$

Donde el ingreso gravable es:

$$
I G_{h}=\sum_{l=1}^{17}\left(P L_{l} * L F_{h, l}\right)+P K * K F
$$




\section{Cuadro 3 \\ Identificadores del modelo}

\begin{tabular}{|c|c|c|}
\hline Identificador & Número & Agente \\
\hline \multirow{10}{*}{$\mathrm{h}$} & 1 & Primer decil de ingreso \\
\hline & 2 & Segundo decil de ingreso \\
\hline & 3 & Tercer decil de ingreso \\
\hline & 4 & Cuarto decil de ingreso \\
\hline & 5 & Quinto decil de ingreso \\
\hline & 6 & Sexto decil de ingreso \\
\hline & 7 & Séptimo decil de ingreso \\
\hline & 8 & Octavo decil de ingreso \\
\hline & 9 & Noveno decil de ingreso \\
\hline & 10 & Décimo decil de ingreso \\
\hline \multirow{2}{*}{$\mathrm{g}$} & 1 & Gobiernos locales, estatal y municipal \\
\hline & 2 & Gobierno federal \\
\hline \multirow{10}{*}{$\mathrm{f}$} & 1 & Alimentos, bebidas y tabaco \\
\hline & 2 & Vestido y calzado \\
\hline & 3 & Vivienda, electricidad, gas, agua y otros combustibles \\
\hline & 4 & Mobiliario, equipo y enseres domésticos \\
\hline & 5 & Sanidad \\
\hline & 6 & Transporte \\
\hline & 7 & Esparcimiento y cultura \\
\hline & 8 & Educación \\
\hline & 9 & Hoteles, cafeterías y restaurantes \\
\hline & 10 & $\begin{array}{l}\text { Bienes y servicios diversos (cuidados personales, comunicaciones, servicios } \\
\text { sociales, financieros y otros servicios) }\end{array}$ \\
\hline \multirow{17}{*}{$\mathrm{i}, \mathrm{j}$} & 1 & Agricultura, ganadería, silvicultura y pesca \\
\hline & 2 & Minería \\
\hline & 3 & Alimentos, bebidas y tabaco \\
\hline & 4 & Indus tria textil \\
\hline & 5 & Industria de la madera \\
\hline & 6 & Industria del papel \\
\hline & 7 & Industria química \\
\hline & 8 & Productos de Minerales No Metálicos \\
\hline & 9 & Industria Metálica Básica \\
\hline & 10 & Productos Metálicos, Maquinaria y Equipo \\
\hline & 11 & Otras Industrias Manufactureras \\
\hline & 12 & Construcción \\
\hline & 13 & Electricidad, gas y agua \\
\hline & 14 & Comercio, restaurantes y hoteles \\
\hline & 15 & Comunicaciones y transportes \\
\hline & 16 & Servicios financieros y alquiler de inmuebles \\
\hline & 17 & Servicios comunales, sociales y personales \\
\hline
\end{tabular}

Revista de Economía - Vol. XXX - Núm. 81 


\section{Cuadro 3}

(continuación)

\begin{tabular}{|c|c|c|}
\hline Identificador & Número & Agente \\
\hline & 1 & Profesionistas \\
\hline & 2 & Técnicos \\
\hline & 3 & Trabajadores de la educación, del arte y deportes \\
\hline & 4 & Funcionarios y directivos de los sectores público, privado y social \\
\hline & 5 & Trabajadores en actividades agrícolas, ganaderas, silvícolas y de caza y pesca \\
\hline & 6 & $\begin{array}{l}\text { Jefes de supervisiones u otros trabajadores de control en la fabricación } \\
\text { artesanal e industrial y en actividades de reparación y mantenimiento }\end{array}$ \\
\hline & 7 & $\begin{array}{l}\text { Artesanos, trabajadores fabriles en la industria de la transformación, y } \\
\text { trabajadores en actividades de reparación y mantenimiento }\end{array}$ \\
\hline & 8 & $\begin{array}{l}\text { Operadores de maquinaria fija de movimiento continuo y equipos en el proceso } \\
\text { de fabricación industrial }\end{array}$ \\
\hline \multirow[t]{9}{*}{1} & 9 & $\begin{array}{l}\text { Ayudantes, peones y similares en el proceso de la fabricación artesanal e } \\
\text { industrial y en actividades de reparación y mantenimiento }\end{array}$ \\
\hline & 10 & $\begin{array}{l}\text { Conductores y ayudantes de conductores de maquinaria móvil y medios de } \\
\text { transporte }\end{array}$ \\
\hline & 11 & $\begin{array}{l}\text { Jefes de departamento, coordinadores, y supervisores en actividades } \\
\text { adminis trativas y de servicios }\end{array}$ \\
\hline & 12 & Trabajadores de apoyo en actividades adminis trativas \\
\hline & 13 & Comerciantes, empleados de comercio y agentes de ventas \\
\hline & 14 & Vendedores ambulantes y trabajadores ambulantes en servicios \\
\hline & 15 & Trabajadores en servicios personales en abastecimientos \\
\hline & 16 & Trabajadores en servicios domésticos \\
\hline & 17 & Trabajadores en servicios de protección y vigilancia y fuerzas armadas \\
\hline
\end{tabular}

\section{Fuente: Elaboración propia}




\subsection{Empresas}

El modelo considera 17 empresas o sectores económicos, se asume que cada una produce un bien homogéneo mediante una función de producción anidada en tres niveles.

En la primera etapa, el valor agregado del sector $j$ se genera al combinar factores primarios (trabajo y capital). La empresa $j$ elije cuánto demandar de cada tipo de ocupación $\left(L A_{l, j}\right)$ y de capital $\left(K A_{j}\right)$ minimizando el costo de generar valor agregado $(V)$ sujeto a la restricción tecnológica, tomando como dados los precios de los tipos de trabajo $P L_{l}$ y del capital $P K$ :

$$
\begin{gathered}
\text { Min } \sum_{l=1}^{17} P L_{l} * L A_{l, j}+P K * K A_{j} \\
\text { s. a. } V_{j}=D_{j} K A_{j}^{\gamma_{k, j}} \prod_{l=1}^{17} L A_{l, j}^{\gamma_{l, j}} \\
\text { para } j=1,2,3, \ldots, 17 ; l=1,2,3, \ldots, 17 ; y k=1 .
\end{gathered}
$$

Donde $D_{j}$ es el coeficiente de la función del valor agregado del sector $j$.

El valor agregado se genera combinando trabajo y capital, mediante una tecnología Cobb Douglas con rendimientos constantes a escala, tal que: $\gamma_{k, j}+\sum_{l=1}^{17} \gamma_{l, j}=1$. De esta manera se permite sustitución entre los insumos primarios, es decir, los 17 tipos de ocupación y el capital $\left(L A_{l, j}\right.$ y $\left.K A_{j}\right)$. Como resultado de este proceso se obtienen las demandas derivadas de factores, en función del nivel de valor agregado y de los precios relativos de los tipos de trabajo y del capital ${ }^{3}$ :

$$
L A_{l, j}=\left(\frac{\gamma_{l, j} * V_{j}}{P L_{l}}\right) * P V_{j}
$$

\footnotetext{
${ }^{3}$ En la sección 5, con el objeto de simplificar los resultados, las demandas derivadas por tipo de trabajo $l$ y por sector $j$ se presentan de forma agregada para los tipos de ocupaciones: (8b) $L A_{l \cdot}=\sum_{j=1}^{17} L A_{l, j}$

Es decir, no se presentan las demandas de los 17 tipos de ocupaciones que realizan los 17 sectores económicos, sino únicamente las demandas agregadas por tipo de ocupación.
} 


$$
K A_{j}=\left(\frac{\gamma_{k, j} * V_{j}}{P K}\right) * P V_{j}
$$

En la segunda etapa, se determina la producción regional del sector $j$. La empresa $j$ decide cuánto demandar de bienes intermedios provistos por ella misma y/u otras empresas $\left(z_{i, j}\right)$, así como de valor agregado $\left(V_{j}\right)$, a través de minimizar el costo de la producción de la región sujeto a la restricción tecnológica tomando como dados los precios de los bienes intermedios $\left(P Y_{i}\right)$ y del valor agregado $\left(P V_{j}\right)$ :

$$
\begin{gathered}
\operatorname{Min} \sum_{i=1}^{17} P Y_{i} * z_{i, j}+P V_{j} * V_{j} \\
\text { s.a. } Y_{j}=\operatorname{Min}\left\{\frac{z_{1, j}}{a_{1, j}}, \ldots, \frac{z_{i, j}}{a_{i, j}}, \ldots, \frac{z_{17, j}}{a_{17, j}}, \frac{V_{j}}{v_{j}}\right\} \\
\text { para } j=1,2,3, \ldots, 17 ; \text { e } i=1,2,3, \ldots, 17 .
\end{gathered}
$$

Donde la producción del sector $j$ en la región $\left(Y_{j}\right)$ utiliza bienes intermedios y valor agregado en proporciones fijas mediante una función de tipo Leontief; tal que $a_{i, j}$ es el requerimiento del insumo vendido por el sector $i$ para producir una unidad del bien del sector $j$ y $v_{j}$ es la cantidad necesaria de valor agregado por unidad de producto del sector $j$. En este sentido, las demandas de bienes intermedios y de valor agregado dependen únicamente del nivel de producción regional o interna, no se ven afectadas por los precios relativos ya que son complementarios:

$$
\begin{aligned}
z_{i, j} & =a_{i, j} * Y_{j} \\
V_{j} & =v_{j} * Y_{j}
\end{aligned}
$$

Finalmente, se obtiene la oferta total del bien provisto por el sector $j$. La empresa elige el nivel de producción regional $\left(Y_{j}\right)$ y externa $\left(M_{j}\right)$, que minimiza el costo total de ofertar el bien dentro de la región, sin importar su región y/o país de origen, sujeto a la restricción tecnológica, tomando como dados los precios de la producción regional $\left(P Y_{j}\right)$ y externa $\left(P m_{j}\right)$ :

$$
\operatorname{Min} P Y_{j} * Y_{j}+P m_{j} * M_{j}
$$




$$
\begin{gathered}
\text { s.a. } Q_{j}=A Q_{j} Y_{j}^{b_{j}} M_{j}^{1-b_{j}} \\
\text { para } j=1,2,3, \ldots, 17 .
\end{gathered}
$$

Donde $A Q_{j}$ es el coeficiente de la función de producción total del sector $j$. Se establece el supuesto de Armington (1969), el cual implica que bienes de distintas regiones y/o países son sustitutos imperfectos, lo que explica que las regiones y/o países produzcan e importen los mismos bienes pero con cualidades distintas. De esta manera, la oferta total del sector $j$ en la región $\left(Q_{j}\right)$ se obtiene al combinar producción regional $\left(Y_{j}\right)$ e importaciones $\left(M_{j}\right)$, suponiendo una función Cobb Douglas con rendimientos constantes a escala, lo cual permite cierto grado de sustitución entre ellas (a este tipo de función se le conoce como Agregador de Armington). Así, las demandas de producción regional e importaciones están en función del nivel de oferta total y de sus precios relativos:

$$
\begin{gathered}
Y_{j}=\left(\frac{Q_{j}}{A Q_{j}}\right) *\left[\frac{b_{j} * P m_{j}}{\left(1-b_{j}\right) * P Y_{j}}\right]^{\left(1-b_{j}\right)} \\
M_{j}=\left(\frac{Q_{j}}{A Q_{j}}\right) *\left[\frac{\left(1-b_{j}\right) * P Y_{j}}{b_{j} * P m_{j}}\right]^{b_{j}}
\end{gathered}
$$

En el caso de los bienes finales de consumo $\left(c_{f}\right)$ supondremos que las empresas eligen cuánto demandar de productos del sector $i$ para el bien final $f\left(q_{i, f}\right)$, minimizando el costo de ofrecer bienes de consumo sujeto a la restricción tecnológica, la cual es una función del tipo Leontief tal que:

$$
\begin{gathered}
\operatorname{Min} \sum_{i=1}^{17} P Q_{i} * q_{i, f} \\
\text { s.a.c } c_{f}=\operatorname{Min}\left\{\frac{q_{1, f}}{\varphi_{1, f}}, \ldots, \frac{q_{i, f}}{\varphi_{i, f}}, \ldots, \frac{q_{17, f}}{\varphi_{17, f}}\right\} \\
\text { para } i=1,2,3, \ldots, 17 ; y f=1,2,3, \ldots, 10 .
\end{gathered}
$$


Donde $\varphi_{i, f}$ es el requerimiento del sector $i$ por unidad del bien de consumo final $f$.

De esta manera, la demanda de bienes provistos por el sector $i$ para generar el bien final $f$ es:

$$
q_{i, f}=\varphi_{i, f} * c_{f}
$$

\subsection{Bien de inversión}

En esta economía se cuenta sólo con un bien de inversión, tanto privado como público, el cual es un agregado de bienes provistos por los sectores económicos (IDA). Se asume que (IDA) es una función de proporciones fijas, lo que significa que se requiere una proporción fija de bienes del sector $i\left(\mu_{i}\right)$ para ser destinada a la inversión, tal que:

$$
I D A=\operatorname{Min}\left\{\frac{I_{1}}{\mu_{1}}, \ldots, \frac{I_{i}}{\mu_{i}}, \ldots, \frac{I_{17}}{\mu_{17}}\right\}
$$

De esta manera, se decide cuánto demandar de cada bien del sector $i$ para invertir $\left(I_{i}\right)$, minimizando el gasto total en inversión dados los precios de dichos bienes $\left(P Q_{i}\right)$, sujeto al nivel de demanda agregada de inversión (IDA). El proceso de optimización es:

$$
\begin{gathered}
\operatorname{Min} \sum_{i=1}^{17} P Q_{i} * I_{i} \\
\text { s.a.IDA }=\operatorname{Min}\left\{\frac{I_{1}}{\mu_{1}}, \ldots, \frac{I_{i}}{\mu_{i}}, \ldots, \frac{I_{17}}{\mu_{17}}\right\} \\
\text { para } i=1,2,3, \ldots, 17 .
\end{gathered}
$$

Como resultado, la demanda de productos del sector $i$ para la inversión es igual a:

$$
I_{i}=\mu_{i} * I D A
$$




\subsection{Gasto público en bienes y servicios}

Se especifica la conducta de dos niveles de gobierno, los locales y el federal, el primero es el único que gasta en bienes intermedios y factores productivos, con la finalidad de proporcionar bienes y servicios públicos en el estado. El gobierno local asigna su gasto al seguir un proceso de optimización en dos niveles. En el primer nivel decide cuánto demandar de cada tipo de ocupación $\left(L G_{l}\right)$ y de capital $(K G)$, al minimizar su gasto en valor agregado $(V G)$ :

$$
\begin{gathered}
\operatorname{Min} \sum_{l=1}^{17} P L_{l} * L G_{l}+P K * K G \\
\text { s.a. } V G=E G(K G)^{\gamma g_{k}} \prod_{l=1}^{17} L G_{l}^{\gamma g_{l}} \\
\quad \text { para } l=1,2,3, \ldots, 17 ; y k=1 .
\end{gathered}
$$

Donde $E G$ es la constante de la función de valor agregado del gobierno. Como resultado se obtienen las demandas de los tipos de ocupación y del capital, en función del valor agregado y de los precios relativos de los factores productivos:

$$
\begin{gathered}
L G_{l}=\left(\frac{\gamma g_{l} * V G}{P L_{l}}\right) * P V G \\
K G=\left(\frac{\gamma g_{k} * V G}{P K}\right) * P V G
\end{gathered}
$$

Donde $P V G$ es el precio del valor agregado de los bienes y servicios públicos locales.

En el siguiente nivel, los gobiernos locales deciden cuánto demandar de bienes productivos $\left(z g_{i}\right)$, así como de valor agregado $(V G)$, a través de minimizar el gasto, tomando como dados los precios de los bienes $\left(P Q_{i}\right)$ y del valor agregado $(P V G)$ : 


$$
\begin{gathered}
\text { Min } \sum_{j=1}^{17} P Q_{i} * z g_{i}+P V G * V G \\
\text { s. } a . B G=\operatorname{Min}\left\{\frac{z g_{1}}{a g_{1}}, \ldots, \frac{z g_{i}}{a g_{i}}, \ldots, \frac{z g_{17}}{a g_{17}}, \frac{V G}{v g}\right\} \\
\text { para } i=1,2,3, \ldots, 17 .
\end{gathered}
$$

Se asume una función de proporciones fijas, que implica que se gasta un porcentaje constante en los sectores económicos $\left(a g_{i}\right)$ y valor agregado $(v g)$ tal que, las demandas son:

$$
\begin{aligned}
& z g_{i}=a g_{i} * B G \\
& V G=v g * B G
\end{aligned}
$$

\subsection{Precios}

En el modelo se asume competencia perfecta, es decir, que todos los agentes del modelo toman sus decisiones considerando que no pueden afectar los precios de los productos y de los factores productivos. Por lo anterior, los precios relacionados con el lado del consumo igualan al gasto unitario, mientras que los precios del lado de la producción igualan a los costos unitarios. En este sentido, los precios de equilibrio resultan de sustituir los óptimos en las respectivas funciones de gastos y costos unitarios.

Los precios del valor agregado de los bienes privados $\left(P V_{j}\right)$ y de los bienes y servicios públicos $(P V G)$ se obtienen al sustituir las demandas de factores primarios en las respectivas funciones de costo unitario de generar valor agregado:

$$
\begin{aligned}
& P V_{j}=\left(\frac{1}{D_{j}}\right) *\left[\prod_{l=1}^{17}\left(\frac{P L_{l}}{\gamma_{l, j}}\right)^{\gamma_{l, j}}\right] *\left(\frac{P K}{\gamma_{k, j}}\right)^{\gamma_{k, j}} \\
& V G=\left(\frac{1}{E G}\right) *\left[\prod_{l=l 1}^{l 17}\left(\frac{P L_{l}}{\gamma g_{l}}\right)^{\gamma g_{l}}\right] *\left(\frac{P K}{\gamma g_{k}}\right)^{\gamma g_{k}}
\end{aligned}
$$


El precio de la producción regional sigue la especificación de la ecuación formadora de precios de un modelo lineal, debido a que se asumió una función de producción de tipo Leontief:

$$
P Y_{j}=\left(1+\sum_{g=1}^{2} T P_{g, j}\right) *\left(\sum_{i=1}^{17} a_{i j} * P Y_{j}+v_{j} * P V_{j}\right)
$$

Donde $T P_{g, j}$ son los impuestos sobre la producción netos de subsidios que carga el nivel de gobierno $g$ en el sector $j .{ }^{4}$ Por otro lado, el precio de la producción externa $\left(P m_{j}\right)$ se considera exógeno e igual a 1.

El precio de la producción total del sector $j$ resulta de introducir los niveles de equilibrio de la producción regional e importada en el costo unitario de producción:

$$
P Q_{j}=\left(\frac{1}{A Q_{j}}\right)\left(\frac{P m_{j}}{1-b_{j}}\right)^{\left(1-b_{j}\right)}\left(\frac{P Y_{j}}{b_{j}}\right)^{b_{j}}
$$

El precio de los bienes de consumo final $\left(P_{f}\right)$ es un promedio ponderado de los precios de los bienes provistos por los sectores económicos:

$$
P_{f}=\sum_{j=1}^{17}\left(\varphi_{j, f} * P Q_{j}\right)
$$

Mientras que el precio del bien de consumo agregado que enfrenta cada hogar de nivel de ingreso $h$, resulta de introducir los niveles óptimos de consumo familiar en bienes finales dentro del gasto unitario en consumo:

$$
P A C_{h}=\left(\frac{1}{A 0_{h}}\right)\left[\prod_{f=1}^{10}\left(\frac{P_{f}}{\alpha_{f, h}}\right)^{\alpha_{f, h}}\right]
$$

\footnotetext{
${ }^{4}$ Los impuestos sobre la producción netos de subsidios aplicados en el ámbito local que se consideran en el modelo son: el impuesto sobre nóminas, el impuesto sobre hospedaje y el impuesto sobre loterías (los dos últimos se aplican en el sector de servicios comunales, sociales y personales). Mientras que del gobierno federal se incluye el impuesto al valor agregado, el impuesto especial sobre producción y servicios, los aranceles y los subsidios a los sectores de electricidad y agropecuario.
} 
El precio del bien de inversión $P S$ es un promedio ponderado de los precios de bienes provistos por los sectores productivos:

$$
P S=\sum_{j=1}^{17} \mu_{j} * P Q_{j}
$$

Finalmente, el precio agregado de los bienes y servicios públicos está dado por la siguiente expresión:

$$
P B G=\left(1+\sum_{g=1}^{2} T G_{g}\right) *\left[\sum_{j=1}^{17}\left(a g_{j} * P Q_{j}\right)+v g * P V G\right]
$$

Donde $T G_{g}$ es el impuesto que pagan los gobiernos locales al nivel de gobierno $g$.

\subsection{Mercados de factores}

Se supone perfecta movilidad de trabajo y capital entre las actividades productivas, pero no así entre regiones, ni países. En el modelo, tanto las empresas como los gobiernos locales utilizan factores primarios para producir. Como regla de cierre se establece que en el mercado de trabajo el equilibrio se logra vía precios, es decir, que los salarios de los diferentes tipos de ocupación se ajustan para que la oferta y la demanda de los tipos de trabajo se igualen, por lo que existe pleno empleo de recursos. En cuanto al mercado de capital, la renta de capital se supone fija e igual a 1, esto es, como el numerario del modelo. En ambos mercados las ofertas de factores se establecen como fijas o perfectamente inelásticas.

$$
\begin{gathered}
L O_{l}=\sum_{h=1}^{10} L F_{h, l} \\
L A D_{l}=\sum_{j=1}^{17} L A_{l, j}+L G_{l}
\end{gathered}
$$




$$
\begin{gathered}
K O=\sum_{h=1}^{10} K F_{h} \\
K A D=\sum_{j=1}^{17} K A_{j}+K G
\end{gathered}
$$

\subsection{Finanzas gubernamentales}

\subsubsection{Gobiernos locales}

Los gobiernos locales se abastecen de la recaudación de impuestos al ingreso de las familias $\left(R T H_{1}\right)$, a los sectores productivos y al mismo gobierno local $\left(R T P_{1}\right)$; así como de transferencias provenientes del gobierno federal $\left(T R G_{12}\right)$. Tal que, el ingreso total de este nivel de gobierno $\left(\operatorname{Ing} G_{1}\right)$ es:

$$
\text { Ing } G_{1}=R T H_{1}+R T P_{1}+T R G_{12}
$$

Dónde:

$$
\begin{gathered}
R T H_{1}=\sum_{h=1}^{10} T H_{1, h} * I G_{h} \\
R T P_{1}=\sum_{j=1}^{17} T P_{1, j} *\left(\frac{P Y_{j}}{1+\sum_{g=1}^{2} T P_{g, j}}\right) * Y_{j}+T G_{1} *\left(\frac{P B G}{1+\sum_{g=1}^{2} T G_{g}}\right) * B G
\end{gathered}
$$

Con estos ingresos, los gobiernos locales compran bienes intermedios y contratan factores productivos $(B G)$, además de que otorgan transferencias directas a los hogares $\left(T R_{1}\right)$, por lo que su gasto total $\left(G G_{1}\right)$ está dado por la siguiente expresión:

$$
G G_{1}=P B G_{1} * B G+T R_{1}
$$


Dónde:

$$
T R_{1} \cdot=\sum_{h=1}^{10} T R_{1, h}=\sum_{h=1}^{10}\left(P A M_{h}+D I S_{h}+B Y A_{h}\right)
$$

De esta manera, el saldo presupuestal de los gobiernos locales $\left(S G_{1}\right)$ es:

$$
S G_{1}=I n g G_{1}-G G_{1}
$$

\subsubsection{Gobierno federal}

Este nivel de gobierno deriva recursos de la recaudación del impuesto sobre la renta de los hogares $\left(R T H_{2}\right)$ y sobre la actividad productiva de las empresas y de los gobiernos locales $\left(R T P_{2}\right)$, por lo que el ingreso total $\left(\operatorname{Ing} G_{2}\right)$ es:

$$
\operatorname{Ing} G_{2}=\mathrm{RTH}_{2}+\mathrm{RTP}_{2}
$$

Dónde:

$$
\begin{gathered}
R T H_{2}=\sum_{h=1}^{10} T H_{2, h} * I G_{h} \\
R T P_{2}=\sum_{j=1}^{17} T P_{2, j} *\left(\frac{P Y_{j}}{1+\sum_{g=1}^{2} T P_{g, j}}\right) * Y_{j}+T G_{2} *\left(\frac{P B G}{1+\sum_{g=1}^{2} T G_{g}}\right) * B G
\end{gathered}
$$

En el modelo, el gobierno federal otorga transferencias directas a los hogares en la región $\left(T R_{2}\right)$, da subsidios al sector agropecuario y al proveedor de energía eléctrica (los cuales entran con signo negativo en los impuestos sobre la producción netos de subsidios) y regresa parte de la recaudación de los impuestos federales a los gobiernos locales vía aportaciones y participaciones $\left(T R G_{12}\right)$. Por lo tanto, el gasto federal ejercido en la región $\left(G G_{2}\right)$ es una variable exógena igual a:

$$
G G_{2}=T R_{2}+T R G_{12}
$$


Dónde:

$$
T R_{2}=\sum_{h=1}^{10} T R_{2, h}
$$

En consecuencia, el saldo presupuestal del gobierno federal en la región $\left(S G_{2}\right)$ es:

$$
S G_{2}=I n g G_{2}-G G_{2}
$$

\subsection{Sector externo}

La decisión del sector externo de cuánto demandar de productos regionales (exportaciones) queda fuera de los agentes participantes en el modelo, como consecuencia de que sólo especificamos la economía regional.

De esta manera, el saldo exterior por cuenta corriente está dado por la siguiente expresión:

$$
\begin{gathered}
S X=-\sum_{j=1}^{17}\left(P Y_{j} * \operatorname{Exp}_{j}-P Q_{j} * M_{j}\right)-\sum_{h=1}^{10} R E M_{h} \\
\text { para } j=1,2,3, \ldots, 17 ; y \text { h } h=1,2,3, \ldots, 10 .
\end{gathered}
$$

La regla de cierre en el sector externo es suponer que las exportaciones son exógenas y que las importaciones y la cuenta corriente son variables.

\subsection{Cuenta agregada de capital}

La oferta agregada de inversión $(I O)$ la determina el ahorro agregado de la economía $(S A)$, el cual proviene de la suma del ahorro de las familias $(S)$, de los dos niveles de gobierno $\left(S G_{1}\right.$ y $\left.S G_{2}\right)$ y del sector externo $(S X)$ :

$$
I O=S .+S G 1+S G 2+S X
$$

Dónde:

$$
S .=\sum_{h=1}^{10} S_{h}
$$


El equilibrio se logra igualándose demanda y oferta de inversión, y el cierre contable del modelo se garantiza cumpliendo con la identidad macroeconómica de inversión agregada igual a ahorro agregado:

$$
I D A=I O=S A
$$

\subsection{Equilibrio}

Además de los cierres para los mercados de factores, el sector externo y la cuenta agregada de capital, el último cierre que completa el modelo consiste en que la oferta de bienes producidos sea igual a la demanda para cada sector:

$$
\begin{gathered}
Q_{i}=q_{i \cdot}+z_{i \cdot}+I_{i}+z g_{i}+E x p_{i} \\
\text { para } i=1,2,3, \ldots, 17
\end{gathered}
$$

Dónde:

$$
\begin{aligned}
& q_{i .}=\sum_{f=1}^{10} q_{i, f} \\
& z_{i \cdot}=\sum_{j=1}^{17} z_{i, j}
\end{aligned}
$$

Los bienes producidos por los sectores son demandados para producir bienes finales $\left(q_{i}\right)$, bienes intermedios $\left(z_{i}\right)$, para invertir $\left(I_{i}\right)$, para proporcionar bienes y servicios públicos $\left(z g_{i}\right)$ y para exportar $\left(\operatorname{Exp}_{i}\right)$.

El equilibrio se define como un vector de precios finales, precios de factores productivos, las asignaciones de consumo y producción, y las recaudaciones tales que los consumidores maximizan su utilidad dado su ingreso disponible, los productores minimizan su costo sujeto a la restricción tecnológica, las recaudaciones impositivas de los dos niveles de gobierno coinciden con los pagos impositivos realizados por los distintos agentes, los mercados se vacían y el saldo exterior y el de los tipos de gobierno se determinan de forma endógena. 


\subsection{Extensiones del modelo}

Ya que el MECG se emplea para analizar una política directamente relacionada con la distribución del ingreso entre los hogares, se estima el coeficiente de Gini tanto para el equilibrio inicial como para el contrafactual, con el fin de medir el cambio en la desigualdad en el ingreso de las familias.

La expresión que se utiliza para calcular el coeficiente de Gini para datos agregados es la siguiente:

$$
\text { C. Gini }=1-\sum_{\mathrm{h}=1}^{10} \mathrm{p}_{\mathrm{h}}\left(\mathrm{Y}_{\mathrm{h}}-\mathrm{Y}_{\mathrm{h}-1}\right)
$$

En donde $\mathrm{p}_{\mathrm{h}}$ representa la proporción de la población en el grupo h; mientras que $\mathrm{Y}_{\mathrm{h}}$ y $\mathrm{Y}_{\mathrm{h}-1}$ representan los ingresos acumulados en los grupos h y $(\mathrm{h}-1)$.

\section{BASE DE DATOS Y CALIBRACIÓN}

Una vez que el modelo ha sido especificado de forma teórica, es necesario asignar valores a los parámetros y a las variables exógenas. El método conocido como calibración es el procedimiento más utilizado para estimar el valor de los parámetros, y requiere de la base de datos conocida como matriz de contabilidad social (MCS).

La MCS utilizada para este trabajo fue elaborada por Chapa, Rangel y Ramírez (2008) y se construyó para la economía del estado de Nuevo León del año 2004 (MCS NL2004). La MCS NL2004 está conformada por las siguientes cuentas: diez tipos de hogares por deciles de ingreso, 17 tipos de ocupaciones, 17 sectores productivos que producen un bien intermedio cada uno, diez bienes finales o de consumo, un tipo de capital, un tipo de sociedades ${ }^{5}$, dos niveles de gobierno (el federal y el estatal y

\footnotetext{
${ }^{5}$ En la MCS NL2004 existe una cuenta que se llama sociedades, la cual representa a las empresas. Éstas reciben el pago del capital y lo distribuyen entre los hogares. Sin embargo, en el diseño del MEGC se empleó el supuesto de que el rendimiento del capital se distribuye directamente entre las familias para simplificar las relaciones entre los agentes de la economía y porque los resultados no se alteran.
} 
municipal), una cuenta agregada de ahorro- inversión y un sector externo que agrega el resto de los estados y el resto del mundo (ver figura 1). ${ }^{6} \mathrm{El}$ sector del gobierno estatal y municipal, a su vez, fue desagregado por el gasto en actividades económicas y factores productivos, así como en las transferencias a los hogares, que son tres: becas y ayudas, el programa de Apoyo directo al adulto mayor y el programa de Apoyo a personas con discapacidad.

Figura 1

Representación esquemática de la MSC NL2004

\begin{tabular}{|c|c|c|c|c|c|c|c|c|c|c|}
\hline & H & SOC & G1 & G2 & S-I & L & K & AE & C & SE \\
\hline H & & & & & & & & & & \\
\hline SOC & & & & & & & & & & \\
\hline G1 & & & & & & & & & & \\
\hline G2 & & & & & & & & & & \\
\hline S-I & & & & & & & & & & \\
\hline L & & & & & & & & & & \\
\hline K & & & & & & & & & & \\
\hline AE & & & & & & & & & & \\
\hline C & & & & & & & & & & \\
\hline SE & & & & & & & & & \\
\hline
\end{tabular}

Fuente: Elaboración propia.

Cabe destacar que Ayala y Chapa (2011) corrigieron el ingreso laboral de la MCS NL2004 al adjudicar un salario al trabajo de los cuenta propia, empleadores y trabajadores familiares sin pago, ya que las remuneraciones del Sistema de cuentas nacionales de México no lo toman en cuenta y por ello están subestimadas.

\footnotetext{
${ }^{6}$ Para la elaboración de la MCS NL2004 no se contó con información confiable para especificar por separado las transacciones de Nuevo León con los demás estados de la república mexicana y con el extranjero.
} 


\section{RESULTADOS}

En esta sección se emplea el modelo de equilibrio general computable construido para el estado de Nuevo León del año 2004, para analizar la importancia del PAM en la economía regional. Se lleva a cabo una simulación en donde se elimina la transferencia otorgada por el programa y estos recursos son utilizados por los gobiernos locales para la compra de bienes intermedios y factores productivos, de modo que la restricción presupuestal de los gobiernos locales se mantiene constante. En un sentido más general, se están estudiando los efectos económicos de que el gobierno devuelva recursos a la sociedad de forma directa, especialmente a un grupo que se concentra entre los hogares más pobres, en lugar de entregarlos en forma de bienes y servicios.

\subsection{Efectos iniciales}

Al suponer que se retira el apoyo directo otorgado por el PAM y se gasta en bienes intermedios y factores productivos, por una parte, se da una caída en el ingreso de las familias, ya que todos los hogares tienen una participación en el programa, especialmente los del primer decil, quienes reciben $81 \%$ de la transferencia. En el cuadro 4 se presenta un resumen de los resultados arrojados por el modelo, agregados y desagregados, los cuales capturan los efectos iniciales y los indirectos. 


\section{Cuadro 4}

Resultados, cambios relativos entre el equilibrio inicial y el contrafactual

\begin{tabular}{|c|c|c|c|c|c|c|c|c|}
\hline & $I D_{h}$ & $C_{h}$ & $S_{h}$ & $I G_{h}$ & $I T_{h}$ & $c_{f}$ & $P A C_{h}$ & $P_{f}$ \\
\hline 1 & $-2.2825 \%$ & $-2.2965 \%$ & $-2.2893 \%$ & $0.0009 \%$ & $-2.2283 \%$ & $-0.1208 \%$ & $0.0143 \%$ & $0.0036 \%$ \\
\hline 2 & $-0.1332 \%$ & $-0.1483 \%$ & $-0.1401 \%$ & $0.0086 \%$ & $-0.1290 \%$ & $-0.0230 \%$ & $0.0151 \%$ & $0.0032 \%$ \\
\hline 3 & $-0.0643 \%$ & $-0.0799 \%$ & $-0.0712 \%$ & $0.0113 \%$ & $-0.0615 \%$ & $-0.0966 \%$ & $0.0157 \%$ & $0.0208 \%$ \\
\hline 4 & $-0.0139 \%$ & $-0.0295 \%$ & $-0.0207 \%$ & $0.0169 \%$ & $-0.0127 \%$ & $-0.0584 \%$ & $0.0156 \%$ & $60 \%$ \\
\hline 5 & $0.0083 \%$ & $-0.0083 \%$ & $0.0015 \%$ & $0.0286 \%$ & $0.0092 \%$ & $-0.0657 \%$ & $0.0166 \%$ & \\
\hline 6 & $0.0069 \%$ & $-0.0096 \%$ & $0.0001 \%$ & $0.0164 \%$ & $0.0074 \%$ & -0.03 & 0.0 & \\
\hline 7 & $0.0268 \%$ & $0.0093 \%$ & $0.0199 \%$ & $0.0313 \%$ & $0.0271 \%$ & $-0.0238 \%$ & $0.0175 \%$ & 0.04 \\
\hline 8 & $0.0204 \%$ & $0.0025 \%$ & $0.0136 \%$ & $0.0227 \%$ & $0.0206 \%$ & $-0.0424 \%$ & $0.0179 \%$ & \\
\hline 9 & $0.0428 \%$ & $0.0249 \%$ & $0.0359 \%$ & $0.0441 \%$ & $0.0428 \%$ & $0.0195 \%$ & $0.0178 \%$ & $0.0198 \%$ \\
\hline \multirow[t]{2}{*}{10} & $0.0443 \%$ & $0.0237 \%$ & $0.0375 \%$ & $0.0445 \%$ & $0.0443 \%$ & $-0.0208 \%$ & $0.0206 \%$ & $0.0200 \%$ \\
\hline & $Q_{j}$ & $Y_{j}$ & $v_{j}$ & $M_{j}$ & $\operatorname{Exp}_{i}$ & $z_{i}$ & $q_{i}$ & $z g_{i}$ \\
\hline 1 & $-0.0994 \%$ & $-0.0955 \%$ & $-0.0955 \%$ & $-0.1086 \%$ & - & $-0.0889 \%$ & $-0.1206 \%$ & $0.7510 \%$ \\
\hline 2 & -0.0 & -0.0 & -0.0 & $-0.0339 \%$ & - & & & \\
\hline 3 & $-0.0915 \%$ & $-0.0915 \%$ & $-0.0915 \%$ & - & $0.0000 \%$ & $-0.0866 \%$ & -0.12 & 0.7 \\
\hline 4 & -0.02 & -0.02 & $-0.0258 \%$ & $-0.0187 \%$ & - & & & \\
\hline 5 & $-0.0642 \%$ & $-0.0659 \%$ & $-0.0659 \%$ & $-0.0620 \%$ & - & $-0.0595 \%$ & $-0.0584 \%$ & 0.75 \\
\hline 6 & -0.02 & $-0.0352 \%$ & $-0.0352 \%$ & $-0.0066 \%$ & - & $239 \%$ & -0.0 & \\
\hline 7 & $-0.0319 \%$ & $-0.0358 \%$ & $-0.0358 \%$ & $-0.0253 \%$ & - & $-0.0287 \%$ & $-0.0376 \%$ & \\
\hline 8 & $-0.0486 \%$ & $-0.0486 \%$ & $-0.0486 \%$ & - & $0.0000 \%$ & $-0.0510 \%$ & $-0.0584 \%$ & 0.75 \\
\hline 9 & -0.0 & -0.0 & & - & $00 \%$ & & & \\
\hline 10 & $-0.0493 \%$ & $-0.0551 \%$ & $-0.0551 \%$ & $-0.0439 \%$ & - & -0.029 & -0.0 & \\
\hline 11 & -0.01 & $-0.0171 \%$ & $-0.0171 \%$ & $-0.0101 \%$ & - & $-0.0082 \%$ & $-0.0544 \%$ & $10 \%$ \\
\hline 12 & $-0.0687 \%$ & $-0.0687 \%$ & $-0.0687 \%$ & - & - & & & \\
\hline 13 & -0.03 & $-0.0328 \%$ & -0.03 & - & $0.0000 \%$ & $-0.0341 \%$ & $-0.0966 \%$ & $0.7510 \%$ \\
\hline 14 & $-0.0036 \%$ & $-0.0036 \%$ & -0.00 & - & & -0.0 & & \\
\hline 15 & $-0.0157 \%$ & $-0.0157 \%$ & $-0.0157 \%$ & - & $0.0000 \%$ & $-0.0213 \%$ & $-0.0287 \%$ & $10 \%$ \\
\hline 16 & $87 \%$ & $-0.0487 \%$ & -0.04 & - & & -0.0 & -0.0 & \\
\hline \multirow[t]{2}{*}{17} & $0.0247 \%$ & $0.0247 \%$ & $0.0247 \%$ & - & $0.0000 \%$ & $-0.0091 \%$ & $-0.0461 \%$ & $0.7510 \%$ \\
\hline & $I_{i}$ & $K A_{j}$ & $L A E_{I}$ & $L G_{1}$ & $P Q_{j}$ & $P Y_{j}$ & $P V_{j}$ & $P L_{I}$ \\
\hline 1 & $-0.0961 \%$ & $-0.1271 \%$ & $0.0000 \%$ & - & $-0.0092 \%$ & $-0.0131 \%$ & $-0.0316 \%$ & $0.0461 \%$ \\
\hline 2 & -0.09 & -0.04 & & - & & & & \\
\hline 3 & $-0.0961 \%$ & $-0.0895 \%$ & -0.31 & $0.6814 \%$ & $59 \%$ & & $20 \%$ & \\
\hline 4 & -0.0 & -0.0 & -0.2 & $0.8173 \%$ & & & & \\
\hline 5 & $-0.0961 \%$ & $-0.0696 \%$ & $0.0000 \%$ & - & $0.0023 \%$ & $0.0039 \%$ & $-0.0037 \%$ & -0.1 \\
\hline 6 & $-0.0961 \%$ & $0.0093 \%$ & 0.0 & - & & & & \\
\hline 7 & $-0.0961 \%$ & $-0.0288 \%$ & $0.0000 \%$ & - & $0.0066 \%$ & $0.0105 \%$ & $0.0070 \%$ & -0.000 \\
\hline 8 & $-0.0961 \%$ & $-0.0513 \%$ & $0.0000 \%$ & - & $.0059 \%$ & $0.0059 \%$ & $-0.0027 \%$ & -0.03 \\
\hline 9 & $-0.0961 \%$ & $-0.0444 \%$ & $0.0000 \%$ & - & & 0.007 & -0.0 & -0.0 \\
\hline 10 & $-0.0961 \%$ & $-0.0441 \%$ & $0.0000 \%$ & - & $0.0054 \%$ & $0.0112 \%$ & $0.0110 \%$ & -0.00 \\
\hline 11 & -0. & -0.0 & & - & & & & \\
\hline 12 & $-0.0961 \%$ & $-0.0606 \%$ & $0.0000 \%$ & - & $0.0093 \%$ & $0.0093 \%$ & $0.0081 \%$ & $0.0227 \%$ \\
\hline 13 & - & -0.0307 & & - & & $9 \%$ & $20 \%$ & 0.0 \\
\hline 14 & - & $0.0117 \%$ & $0.0000 \%$ & - & $0.0198 \%$ & $0.0198 \%$ & $0.0153 \%$ & \\
\hline 15 & - & $-0.0029 \%$ & $0.0000 \%$ & - & $0.0146 \%$ & $0.0146 \%$ & $0.0127 \%$ & $0.0407 \%$ \\
\hline 16 & - & $-0.0298 \%$ & $0.0000 \%$ & - & $0.0209 \%$ & $0.0209 \%$ & $0.0189 \%$ & $0.0886 \%$ \\
\hline 17 & $-0.0961 \%$ & $0.0966 \%$ & $0.0000 \%$ & - & $0.0516 \%$ & $0.0516 \%$ & $0.0719 \%$ & $0.0613 \%$ \\
\hline$R T H_{1}$ & $R T P_{1}$ & $\operatorname{lng} G_{1}$ & $S G_{1}$ & $K G$ & $V G$ & $B G$ & PVG & $P B G_{1}$ \\
\hline $0.0370 \%$ & $0.4530 \%$ & $0.0676 \%$ & $0.4994 \%$ & $1.0901 \%$ & $0.7510 \%$ & $0.7510 \%$ & $0.3365 \%$ & $0.3365 \%$ \\
\hline$R \mathrm{TH}_{2}$ & $R T P_{2}$ & $\operatorname{lng} G_{2}$ & $S G_{2}$ & ID. & $c$ & $S$. & IG. & $I T$ \\
\hline $0.0372 \%$ & $-0.0098 \%$ & $0.0052 \%$ & $0.0070 \%$ & $-0.0198 \%$ & $-0.0549 \%$ & $-0.0005 \%$ & $0.0347 \%$ & $-0.0160 \%$ \\
\hline$Q$. & $Y$ & $V$. & $M$. & $s X$ & IDA & $P S$ & $P K$ & C. Gini \\
\hline$-0.0287 \%$ & $-0.0265 \%$ & $-0.0188 \%$ & $-0.0406 \%$ & $-169.48^{*}$ & $-0.0961 \%$ & $0.0069 \%$ & $0.0000 \%$ & $0.0014 \%$ \\
\hline
\end{tabular}

Nota. * Cambio en millones de pesos. Fuente: Elaboración propia.

Julio a Diciembre de 2013 - Págs: 89-128 
La disminución del ingreso disponible de los hogares $\left(I D_{h}\right)$ afecta tanto su consumo $\left(C_{h}\right)$ como su ahorro $\left(S_{h}\right)^{7}$.

La caída en el consumo de las familias se traduce en una disminución de la demanda de bienes finales $\left(c_{f}\right)$, especialmente para Alimentos, bebidas y tabaco $\left(c_{1}\right), 0.1208 \%$, y Vivienda, gas, agua y otros combustibles $\left(c_{3}\right) 0.0966 \%$, que son los bienes en que más gastan los primeros deciles.

También la demanda de bienes intermedios, que se utilizan para producir bienes finales $\left(q_{i}\right)$, se reduce para la mayoría de los sectores. Disminuye en mayor medida la demanda de los sectores que se emplean intensivamente en la producción de los bienes finales $c_{1}$ y $c_{3}$, como: agricultura, ganadería, silvicultura y pesca $\left(q_{1}\right)$, que cae $0.1208 \%$; alimentos, bebidas y tabaco $\left(q_{3}\right), 0.1208 \%$; minería $\left(q_{2}\right), 0.1208 \%$, debido a que se incluye la extracción de petróleo y gas; Industrias metálicas básicas, $0.0966 \%$, ya que los metales procesados en este sector, como el hierro, el acero y el aluminio tienen múltiples usos, por ejemplo, el cableado en las viviendas o el embalaje en la industria alimentaria y el sector electricidad, gas y agua $\left(q_{13}\right) 0.0966 \%$, entre otros.

La producción de bienes intermedios, a su vez, requiere de factores productivos, cuyas demandas derivadas son presionadas a la baja, pero como uno de los supuestos del modelo es que la oferta se mantiene fija, los precios son los que se ajustan disminuyendo para equilibrar los mercados.

Por el lado de los gobiernos locales, éstos gastan en bienes intermedios provistos por 16 sectores $^{8}$, especialmente del sector de servicios comunales, sociales y personales $\left(z g_{17}\right)$, así como en capital $(K G)$ y dos tipos de trabajo, trabajadores de la educación $\left(L G_{3}\right)$ y funcionarios y directivos; por

\footnotetext{
${ }^{7}$ El modelo reporta magnitudes muy similares para estas variables según el decil de ingreso, así como para las demandas de cada bien por tipo de hogar $\left(c_{f, h}\right)$, debido a las formas funcionales utilizadas.

${ }^{8}$ A pesar de que existen 17 sectores en el modelo, los gobiernos locales sólo gastan en 16 sectores. No gastan en AE12, que es el sector de la construcción. Es así porque en el diseño de la MCS NL2004 el gasto que realizan los gobiernos locales en el sector de la construcción se asignó como parte de la inversión que se lleva a cabo en el estado, es decir, la cuenta de inversión incluye la inversión pública y la privada.
} 
lo que incrementa la demanda de todos ellos. ${ }^{9}$ La demanda de bienes intermedios aumenta $0.7510 \%$ para todos los sectores, mientras que los precios de los factores productivos son presionados a subir.

\subsection{Efectos finales sobre la producción, los precios y los hogares}

Los efectos directos en las demandas de bienes intermedios, por el lado de las familias y del gobierno, a su vez afectan las transacciones de cada sector con los demás sectores, de modo que las demandas interindustriales caen para todas las actividades económicas $\left(z_{i}\right)$.

Al final, el efecto neto en la producción tanto doméstica como total es de una caída para casi todos los sectores. Los sectores más afectados son alimentos, bebidas y tabaco $\left(Q_{3}\right)$ cuya oferta cae $0.0915 \%$, ya que, como se mencionó anteriormente, es un insumo importante para el bien final $c_{1}$; pero la mayor caída la presenta agricultura, ganadería, silvicultura y pesca $\left(Q_{1}\right)$ que, además de ser un insumo para $c_{1}$, tiene una relación intersectorial muy fuerte con alimentos, bebidas y tabaco. Por otro lado, la oferta de servicios comunales, sociales y personales $\left(Q_{17}\right)$ es la única en presentar un incremento, porque predomina el efecto del aumento en el gasto del gobierno al ser el bien que más utiliza en términos absolutos. En el agregado, pesa más la caída en la demanda de los hogares y la producción $(Q)$ disminuye 0.0286 por ciento.

Cabe destacar que el efecto negativo en la mayoría de los sectores se refuerza indirectamente por los resultados en la inversión, como se explicará más adelante. Los efectos de la caída en la inversión se observan principalmente en el sector de la construcción $\left(Q_{12}\right)$, que disminuye su producción $0.0687 \%$; en la industria de la madera $\left(Q_{5}\right), 0.0642 \%$ ya que su comprador principal es precisamente la construcción y en productos minerales no metálicos $\left(Q_{8}\right)$ que cae $0.0486 \%$ debido a que el sector incluye la fabricación de cemento, concreto, cal, yeso, entre otros productos.

\footnotetext{
${ }^{9} \mathrm{Si}$ se dejan de lado las transferencias, los gobiernos locales distribuyen su gasto de la siguiente forma: $25.20 \%$ en Trabajadores de la educación, $18.36 \%$ en Funcionarios y directivos, $1.59 \%$ en capital, $39.74 \%$ en servicios comunales, sociales y personales, $7.26 \%$ en los demás sectores económicos y $7.86 \%$ en impuestos.
} 
En cuanto a los factores productivos, una parte de los trabajadores de la educación, funcionarios y directivos y del capital se trasladan del sector privado al sector público, mientras que los trabajos y capital que se quedan en las empresas se mueven al sector de servicios comunales, sociales y personales.

En lo que respecta al sistema de precios es importante mencionar que los precios relativos de los factores productivos son los que forman la base del sistema. Como era de esperarse, el salario relativo de los trabajadores de actividades agrícolas $\left(P L_{5}\right)$ es el que presenta la mayor disminución, $0.1179 \%$, debido a que es el tipo de ocupación que se emplea intensivamente para la producción de agricultura, ganadería, silvicultura y pesca $\left(Q_{1}\right)$. También los salarios de las ocupaciones relacionadas con la fabricación artesanal e industrial, reparación y mantenimiento, operadores de maquinaria y conductores, se mueven a la baja $\left(P L_{6}\right.$ a $\left.P L_{10}\right)$, debido a que, en conjunto, representan una participación importante de los factores empleados en la producción de la mayoría de los sectores, desde la Minería $\left(Q_{2}\right)$ hasta electricidad, gas y agua $\left(Q_{13}\right)$. Por otro lado, los salarios de los trabajadores de la educación $\left(P L_{3}\right)$ y los funcionarios y directivos $\left(P L_{4}\right)$ son los que más se incrementan, $0.4059 \%$ y $0.2706 \%$, respectivamente, por la presión en la demanda que ejerce sobre ellos el gobierno estatal.

Los precios de los bienes intermedios, por su parte, se ven afectados por los cambios en la demanda del bien en cuestión y de los bienes relacionados, así como por los cambios en los salarios de los trabajadores que ocupan como insumos. El precio del bien producido por el sector agricultura, ganadería, silvicultura y pesca $\left(P Q_{1}\right)$ es el único que presenta una baja, $0.0092 \%$, tanto por la caída en la producción de este bien como por la disminución en el salario de los trabajadores de actividades agrícolas $\left(P L_{5}\right)$, factor en el cual es intensivo. Por otro lado, el precio del bien intermedio del sector servicios comunales, sociales y personales $\left(P Q_{17}\right)$ es el que presenta el incremento más significativo, $0.0516 \%$, debido al efecto inicial y por el aumento en el salario de los Trabajadores de la educación $\left(P L_{3}\right)$, que es la ocupación que más utiliza.

Sobre los precios de los bienes finales, todos se incrementan ligeramente. Los cambios mayores son para los precios de los bienes 
Esparcimiento y cultura $\left(P_{7}\right)$ y Educación $\left(P_{8}\right), 0.0405 \%$ y $0.0453 \%$, respectivamente, ya que se componen en gran medida por los insumos provistos por el sector Servicios comunales, sociales y personales. Esto, a su vez, implica que el nivel de precios para cada uno de los consumidores representativos $\left(P A C_{h}\right)$ se incrementa, se observa un efecto progresivo porque aumenta muy poco, $0.0036 \%$, el precio del bien que tiene la mayor participación en el gasto de los hogares pobres, que es alimentos, bebidas y tabaco $\left(P_{1}\right)$.

Al final, el cambio en el ingreso proveniente del pago a los factores productivos $\left(I G_{h}\right)$ es positivo para todos los hogares. Sin embargo, es muy pequeño para los dos deciles más bajos, donde hay una proporción importante de trabajadores en actividades agrícolas $\left(L F_{h, 5}\right)$ y de trabajadores fabriles, operadores de maquinaria, peones, conductores $\left(L F_{h, 7}\right.$ a $\left.L F_{h, 10}\right)$, cuyos salarios caen. Por el contrario, el ingreso proveniente del pago a los factores aumenta en mayor medida para los deciles nueve y diez, en donde predominan los profesionistas $\left(L F_{h, 1}\right)$, trabajadores de la educación $\left(L F_{h, 3}\right)$ y funcionarios $\left(L F_{h, 4}\right) \mathrm{y}$, además, son dueños del capital $(K F)$.

El pequeño incremento en el ingreso gravable de los hogares pobres $\left(I G_{h}\right)$ no alcanza a compensar el retiro de la transferencia, de modo que su ingreso disponible $\left(I D_{h}\right)$ disminuye; mientras que las mayores ganancias se las llevan los deciles nueve y diez, con un aumento de su ingreso disponible de $0.0428 \%$ y $0.0443 \%$, respectivamente, ya que para ellos la transferencia otorgada por el programa no es tan importante.

Dado que del decil siete al diez aumenta el ingreso disponible y el consumo, también se incrementa el consumo en hoteles, cafeterías y restaurantes $\left(c_{9}\right) 0.0195 \%$, por ser un bien que consumen más en términos absolutos y relativos estos hogares. ${ }^{10}$ Asimismo, se observa que para estos hogares el ahorro aumenta más que el consumo en términos relativos, debido a que se esperaría que las familias de ingresos altos ya tuvieran cubiertas sus necesidades básicas de consumo, por lo que el consumo ya no se eleva de forma importante y destinan su excedente de ingreso al ahorro.

\footnotetext{
${ }^{10}$ El incremento en $c_{9}$ aumenta la demanda del bien intermedio comercio, restaurantes y hoteles $\left(q_{14}\right)$, y éste contribuye al aumento de la producción de servicios comunales, sociales y personales $\left(Q_{14}\right)$, por ser su fuente de ingreso más importante.
} 
Al tomar en cuenta los efectos directos e indirectos en la economía, en términos agregados el ingreso disponible (ID) cae $0.0198 \%$, cuando en el equilibrio inicial la transferencia otorgada por el programa representaba únicamente $0.0005 \%$ del ingreso disponible total de las familias. El consumo agregado se reduce $0.0549 \%$, mientras que el ahorro agregado de las familias $(S)$ disminuye $0.0005 \%$ y el ingreso gravable agregado $(I G)$ se incrementa 0.0346 por ciento.

$\mathrm{Al}$ considerar los resultados arrojados por el modelo, el ingreso se redistribuye en la economía hacia una situación más desigual, ya que el coeficiente de Gini aumenta de 0.5057 a 0.5064 , es decir, 0.0014 por ciento.

\subsection{Efectos finales en gobierno, sector externo e inversión}

En cuanto a las finanzas gubernamentales, los gobiernos locales incrementan su ingreso $\left(\operatorname{Ing} G_{1}\right) 0.0676 \%$, vía un aumento en la recaudación total. Se recauda más de todos los hogares $\left(R T H_{1}\right), 0.0370 \%$, porque la tasa impositiva es proporcional al ingreso gravable. También se recauda más de la producción de las empresas y del mismo gobierno local $\left(R T P_{1}\right)$, $0.4530 \%$, especialmente por éste último. Por mantenerse el gasto constante se genera un aumento en el ahorro público de 0.4994 por ciento.

El gobierno federal también incrementa su ingreso $\left(\operatorname{Ing} G_{2}\right)$, $0.0052 \%$, debido a una mayor recaudación en los hogares $\left(\mathrm{RTH}_{2}\right)$, que aumenta $0.0372 \%$, en este caso no se eleva la recaudación de la producción por la caída que se da en el sector privado y porque lo que reciben de los gobiernos locales es una proporción muy pequeña. Con el supuesto de que el gasto permanece constante el saldo $\left(S G_{2}\right)$ es mayor 0.0070 por ciento.

Por otra parte, las compras de Nuevo León al exterior disminuyen en todos los sectores que son importadores netos, especialmente en la Agricultura, ganadería, silvicultura y pesca $\left(M_{1}\right)$, donde caen $-0.1086 \%$ y en el agregado la disminución es de 0.0406 por ciento. Sin embargo, por supuesto del modelo, el ingreso del exterior que entra al estado por medio de las exportaciones permanece constante. El efecto es que en términos relativos la participación del exterior en la economía estatal es mayor, lo que se ve reflejado en un incremento en el déficit de la cuenta corriente $(S X)$. 
Finalmente, se presenta una caída de $0.0961 \%$ en el ahorro agregado de la economía, porque el aumento en el ahorro de los gobiernos $\left(S G_{1}\right.$ y $S G_{2}$ ) no alcanza a compensar la caída en la cuenta corriente $(S X)$ y en el ahorro de las familias $(S$.). Por los supuestos del modelo, el efecto sobre el bien agregado de inversión (IDA) es de la misma magnitud que en el ahorro, de una disminución de $0.0961 \%$, y es igual en cada uno de los sectores.

\section{CONCLUSIONES}

En este trabajo se analizaron los efectos del Programa de apoyo directo al adulto mayor, PAM, sobre la economía del estado de Nuevo León, con un modelo de equilibrio general computable, por ser una de las políticas sociales más importantes de los últimos años en la entidad, en cuanto al alivio de la pobreza. Los resultados del modelo señalan que el PAM ha tenido un impacto positivo en la economía estatal, ha tenido una incidencia positiva en la distribución del ingreso. Además, se encuentra que la introducción del programa, es decir, la entrega de transferencias focalizadas en un grupo de personas, pertenecientes principalmente a la población de más bajos ingresos, ha generado ganancias de eficiencia en la producción agregada y en el ingreso y consumo privado del estado de Nuevo León, contra otorgar los recursos de forma indirecta a través de bienes y servicios.

En cuanto a los efectos sobre las familias, el ingreso se redistribuye de forma más equitativa, ya que aumenta para $40 \%$ de los hogares más pobres y cae para los demás de forma progresiva. La mejora en el ingreso de las familias pobres, además de la transferencia directa, se debe a que, en primer lugar, los sectores más beneficiados como Agricultura y Alimentos, bebidas y tabaco emplean los tipos de ocupaciones que llevan a cabo principalmente las personas pobres, como Trabajadores en actividades agrícolas, ganaderas, silvícolas y de caza y pesca u Operadores de maquinaria fija en el proceso de fabricación industrial. En segundo lugar, estas ocupaciones presentan mejoras en sus salarios, lo que genera que en neto los hogares pobres también obtengan ganancias indirectas.

Desde el punto de vista del impacto económico, los efectos son ligeramente mayores al otorgar el dinero al PAM, que si el gobierno hubiera 
asignado ese monto como lo dicta su estructura de gasto del año 2004. En ese año el gobierno dedicaba un poco más de $40 \%$ de su gasto al pago de las remuneraciones de los Trabajadores de la educación, del arte y deportes, y de los Funcionarios y directivos del sector público; ocupaciones, a su vez, provistas principalmente por personas pertenecientes a los deciles altos de ingreso. Las familias más ricas destinaban una proporción mayor de su ingreso al ahorro y una menor al consumo, contrario a lo que sucede con las familias pobres quienes gastan casi todo su ingreso. Sin embargo, al analizarse todo con un modelo estático, únicamente se vieron reflejados en la producción los efectos del mayor consumo, ya que no fue posible capturar los efectos de corto plazo del mayor ahorro en la acumulación de capital.

Cabe destacar que los resultados fueron de magnitudes pequeñas, ya que también la política analizada fue de baja cuantía, tanto en su participación en el gasto de gobierno $(0.92 \%)$, como en comparación con el tamaño de la economía durante ese año $(0.01 \%)$.Algunos aspectos que se pueden mejorar en el MEGC son: el sistema de demanda de los consumidores, por otro en el que la elasticidad ingreso de la demanda de cada bien no necesariamente sea igual a uno, de modo que las demandas de los distintos bienes no varíen en la mismo magnitud para cada hogar representativo; además, el mercado de trabajo podría especificarse de forma distinta para que incluya una tasa de desempleo.

En cuanto a las limitaciones de este trabajo, por una parte, habría sido más adecuado diferenciar a los tipos de hogares no sólo por su nivel de ingreso, sino también, si se trata de hogares de adultos mayores, para así capturar sus patrones de ingreso y de gasto; en esta línea, una posibilidad sería especificar un modelo con cuatro tipo de familias: los hogares pobres y no pobres, con adultos mayores y sin adultos mayores. Es una línea de investigación a explorar en el futuro. Otra de las limitaciones es que no se cuenta con información para calibrar el modelo con datos más actuales.

Por último, el MEGC elaborado para el estado de Nuevo León cuenta con características similares a los primeros modelos de este tipo realizados a finales de los setenta, es decir, es un modelo estático, de corte neoclásico, con competencia perfecta y formas funcionales sencillas como la Cobb Douglas o de proporciones fijas. Hoy en día los modelos de equi- 
librio general han progresado hasta la elaboración de modelos dinámicos, con procesos estocásticos, con formas funcionales complicadas o estimación de parámetros, mediante métodos econométricos, entre otras características, con la finalidad de reflejar de la mejor manera posible la realidad económica. No obstante, el MECG para Nuevo León, aunque cuenta con limitaciones y oportunidades de perfeccionamiento, constituye uno de los primeros y mayores esfuerzos por aplicar esta metodología en México en nivel regional, con el propósito de ser un instrumento que permita analizar, en un amplio panorama, los efectos de una gran cantidad de políticas que se lleven a cabo en el estado. 


\section{REFERENCIAS}

Ayala E. y J. Chapa. 2011. Adjusting Participation of Remunerations in the Added Value in México. UANL - ITESM.

André, F.J., M.A. Cardenete y E. Velázquez. 2005. "Performing an Environmental Tax Reform in a Regional Economy. A Computable General Equilibrium Approach", The Annals of Regional Science, 39 (2): 375-392.

Armington, P. S. 1969. "A Theory of Demand for Products Distinguished by Place of Production”, IMF Staff Papers, 16: 159-178.

Aguayo, E., J. Chapa, N. Ramírez y E. Rangel. 2009. Análisis de la generación y distribución del ingreso en México del programa Oportunidades a través de un modelo lineal del flujo circular de la renta, en D. Flores, L. Treviño y J. Valero (comps.) La economía mexicana en 19 miradas, Miguel Ángel Porrua, pp. 469-499.

Boyd, R. y K. Dooroodian. 1999. "The Impact of Removing Corn Subsidies in Mexico: A General Equilibrium Assessment", Atlantic Economic Journal, 27(2): 150-169.

Cardenete, M.A. y F. Sancho. 2003. "An Applied General Equilibrium Model to Assess the Impact of National Tax Changes on a Regional Economy", Review of Urban and Regional Development Studies, 15 (1): 55-65.

Cardenete, M.A., F.J. de Miguel y J. Pérez Mayo. 2009. "Effects of the Tax on Retail Sales of some Fuels on a Regional Economy: A Computable General Equilibrium Approach", The Annals of Regional Science, 43 (3): 781-806.

Cardenete, M.A., G.J.D. Hewings y E. Velázquez. 2006. "Precio del agua y relocalización sectorial del recurso en la economía andaluza. Una aproximación desde un modelo de equilibrio general aplicado", Estudios de Economía Aplicada, 24 (3): 1043-1066. 
Coady, D. y R. Harris. 2004. "Evaluating Transfers Programmes within a General Equilibrium Framework", The Economic Journal, 114 (498): 778-779.

Chapa, J. 2003. Análisis de la apertura comercial en México mediante modelos multisectoriales, 1970-93,Universitat de Barcelona, tesis doctoral.

Chapa, J., E. Rangel y N. Ramírez. 2008. Matriz de contabilidad social: Nuevo León, 2004, en Desarrollo social en Nuevo León: reflexiones en torno a la pobreza, desigualdad, migración, bienestar, consumo y grupos vulnerables, Cuaderno del Consejo de Desarrollo Social, núm. 8, pp. 177-229.

De Miguel, F.J. y A. Manresa. 2008. "Removal of Farm Subsidies in a Regional Economy: A Computable General Equilibrium Analysis", Applied Economics, 40 (16): 2109-2120.

Dyer, George, J.E. Taylor y A.Yunez Naude. 1999. “Agricultural Price Policy, Employment, and Migration in a Diversified Rural Economy: A Village-Town CGE Analysis from Mexico", American Journal of Agricultural Economics, 81 (3): 653-662.

Gómez, M., I. Martínez y J. Treviño. 2009. Mapas de pobreza y rezago social, área metropolitana de Monterrey, Consejo de Desarrollo Social de Nuevo León.

González, E. y H. Sobarzo. 1999. "Jalisco y la economía nacional: un modelo de equilibrio general aplicado”, Momento Económico, 104: 35-51.

Hampton S., J.E. Taylor y A. Yúnez Naude. 1999. “Agricultural Policy Reforms and Village Economies: A Computable General-Equilibrium Analysis from Mexico", Journal of Policy Modeling, 2 (4): 453-480.

Harris, R. 2000. Agricultural Policy Reform in Mexico: A Computable General Equilibrium Analysis, The George Washington University, tesis doctoral. 
Kehoe, T.J. y J. Serra Puche. 1983. "A Computational General Equilibrium Model with Endogenous Unemployment: An Analysis of the 1980 Fiscal Reform in Mexico", Journal of Public Economics, 22: 1-26.

1991. "A General Equilibrium Appraisal of Energy Policy in Mexico", Empirical Economics, 16 (1): 71-93.

— y L. Solís. 1986. "A General Equilibrium Model of Domestic Commerce in Mexico", Journal of Policy Modeling, 6: 1-28.

Llop, M. y A. Manresa. 2004. "The General Equilibrium Effects of Social Security Contributions under Alternative Incidence Assumptions", Applied Economics Letters, 11: 847-850.

Martínez Jasso, I. 2006. Memoria del diseño y operación, Cuaderno del Consejo de Desarrollo Social, núm. 1, Nuevo León.

Serra Puche, J. 1979. A Computational General Equilibrium Model for the Mexican Economy: An Analysis of Fiscal Policies. Yale University, tesis doctoral, disponible en ProQuest Dissertations and Theses (PQDT).

Sobarzo, H. 1991. A General Equilibrium Analysis of the Gains from Trade for the Mexican Economy of a North American Free Trade Agreement, Serie documentos de trabajo del CEE, núm. 2.

Sobarzo, H. 1994. A General Equilibrium Analysis of the Gains from Trade for the Mexican Economy of a North American Free Trade Agreement, Federal Reserve Bank of Minneapolis. 